\title{
COVID-19 in Africa
}

\author{
COVID-19 has started to reach Africa, a continent that has in recent decades faced the ongoing HIV/AIDS \\ pandemic and the Ebola epidemic of 2014-2016. Dr Matshidiso Moeti, WHO Regional Director for Africa, talks to \\ Nature Human Behaviour about the African response to COVID-19.
}

\section{What is the current status of COVID-19 across Africa?}

As of 20 March, there are 769 confirmed cases of COVID-19 in 37 countries across Africa, with 15 fatalities. The pandemic has taken longer to reach Africa than other regions, but there has been a significant spike in confirmed cases on the continent within the last 5 days.

It is certainly a concern that we now have 37 countries with reported confirmed cases in Africa when a week ago there were only five countries. But for most of the countries, the confirmed cases are sporadic and are linked to people coming from European countries. So, the top priority for governments in Africa is to contain these few cases by finding their contacts and isolating them as well. It is important not only to have strong surveillance at points of entry, such as airports, but also to improve the capacity to detect cases in health facilities and communities.

WHO has been supporting governments with early detection by providing COVID-19 testing kits, training laboratory technicians and by helping governments to strengthen their surveillance in communities.

There is no time for complacency. WHO will provide support to any country with newly confirmed cases and with critical gaps. We believe that African countries can still change the course of this pandemic.

Why has the pandemic been slower to reach the African continent in comparison to other global regions?

We are still learning a lot about COVID-19 and piecing together evidence-based information on how it will affect different populations. Africa is not the only region to have a relatively low number of confirmed cases of coronavirus. South America is reporting the same kind of sporadic cases linked to travellers from other parts of the world. It's unclear if there is some link between the two regions.

From the evidence so far, the virus that causes COVID-19 can be transmitted in all areas, including areas with hot and humid weather.

What is important to note is that we have not yet seen any clustering of influenza-like illnesses. This reinforces our confidence that the number of cases in Africa remains low,

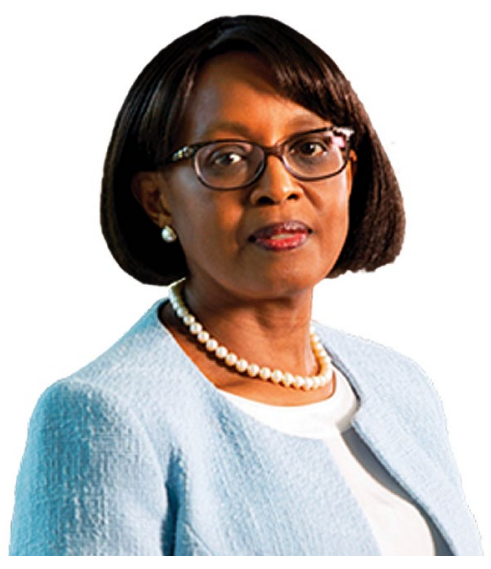

Credit: WHO/AFRO

and we want to take all possible measures to ensure that this continues to be the case. As in all countries, it is possible that some cases of coronavirus in Africa are still being missed, but by and large, we believe that most are being picked up.

\section{Are there lessons learned from the HIV/AIDS pandemic and Ebola epidemic that African countries could draw on at this time to inform their response to the COVID-19 pandemic?}

One of the key lessons learned from previous health crises in Africa is that a rapid, holistic and cohesive response from all arms of government, civil society and local communities is absolutely critical. HIV/AIDS and Ebola both provide compelling case studies of the far-reaching political, socioeconomic and societal ramifications of such crises. As such, they poignantly highlight the need for all sectors to be part of the response to COVID-19. The fatally slow and disjointed initial response to Africa's AIDS epidemic should serve as a stark reminder of past shortcomings. But African countries recently affected by or at risk from Ebola should be better placed to quickly contain the spread of coronavirus. The successful containment of the Democratic Republic of Congo's (DRC) Ebola epidemic should serve as a source of inspiration in this regard. Various preexisting measures and infrastructure from these containment efforts can now serve as valuable assets for containing COVID-19. Previous epidemics have also shown the value of trained community health workers, particularly in remote areas with insufficient formal health facilities. The training and equipment that are already provided by WHO and other partners to health workers for Ebola prevention are equally applicable to coronavirus, as is previous public advice regarding hygiene and social distancing. In summary, preparedness pays off. It is important to note that community engagement is key in effectively responding to the outbreak.

In light of the consequences we've seen so far in response to the actions of other governments worldwide, how do you feel the governments of African countries could move forward?

The most appropriate strategy for countries in the African region remains containment. Containment measures are activities that are aimed at interrupting transmission. This means identifying cases earlier, isolating cases and tracing contacts to prevent or limit person-to-person transmission.

Now that there have been confirmed cases in sub-Saharan Africa, it is especially important that countries put stringent early detection and surveillance measures in place. Our main fear is that countries could develop an unmanageably large caseload that could then easily overwhelm existing health systems that are already dealing with many diseases and challenges. We need-at the same time-to work on worst case scenarios and prepare for mitigation measures. We also have to rethink the health service pathway to ensure continuity of critical services.

What does the risk profile look like for urban vs rural areas of Africa in terms of susceptibility to COVID-19, and are there measures that could be taken that reflect geographic differences in risk?

We do not know enough yet to comment on a risk profile in Africa in terms of susceptibility.

We are still piecing together evidencebased information on how COVID-19 will affect different populations. While in Asia and Europe it appears that older people have been more affected, analysis has also found 
that people with underlying conditions are affected. In Africa there are people with a range of underlying conditions, such as children with malnutrition and people living with HIV, who are not yet accessing treatment. We do not know how the virus will affect them, but they have underlying vulnerabilities that are of concern.

\section{How could African citizens act to mitigate the spread and impact of COVID-19?}

As is the case in all other regions, African citizens should adhere as much as possible to WHO's guidelines on prevention. Personal hygiene, in particular regular handwashing, is vital, as is being informed about COVID-19 and ensuring that any information comes from reliable sources; both the HIV and Ebola epidemics show the potential panic and discrimination that can result from the spread of rumours and misinformation. People should also try to avoid unnecessary travel and take precautions to stay away from crowded areas if over 60 years old or if they have underlying conditions, such as cardiovascular disease, diabetes, chronic respiratory disease or cancer. People in lower-risk categories should be aware of their ability to spread coronavirus to those more vulnerable than them, particularly in contexts where health services are already stretched and where there are high levels of underlying conditions, such as malnutrition or people living with HIV and TB who are not yet accessing treatment. As we have seen with HIV, communities will have to lead prevention and mitigation interventions on the ground. It should be reiterated, however, that many Africans living in poor and overcrowded areas face significant socioeconomic obstacles to such efforts, so the onus is also on all governments and their partners to scale up health services and other critical measures.

Interviewed by Charlotte Payne

Published online: 3 April 2020

https://doi.org/10.1038/s41562-020-0870-5

Competing interests

The authors declare no competing interests. 